\title{
In Silico Analysis of Coding/Noncoding SNPs of Human RETN Gene and Characterization of Their Impact on Resistin Stability and Structure
}

\author{
Lamiae Elkhattabi, ${ }^{1,2}$ Imane Morjane,, ${ }^{1,2}$ Hicham Charoute, ${ }^{1}$ Soumaya Amghar, ${ }^{1}$ \\ Hind Bouafi, ${ }^{1,2}$ Zouhair Elkarhat, ${ }^{1}$ Rachid Saile, ${ }^{2}$ Hassan Rouba, ${ }^{1}$ \\ and Abdelhamid Barakat $\left.{ }^{1}\right)^{1}$ \\ ${ }^{1}$ Laboratoire de Génomique et Génétique Humaine, Institut Pasteur du Maroc, Casablanca, Morocco \\ ${ }^{2}$ Laboratoire de Biologie et Santé, Faculté des Sciences Ben M'Sik, Université Hassan II de Casablanca, Morocco
}

Correspondence should be addressed to Abdelhamid Barakat; hamid.barakat@pasteur.ma

Received 26 October 2018; Revised 25 February 2019; Accepted 1 April 2019; Published 20 May 2019

Academic Editor: Jonathan M. Peterson

Copyright (c) 2019 Lamiae Elkhattabi et al. This is an open access article distributed under the Creative Commons Attribution License, which permits unrestricted use, distribution, and reproduction in any medium, provided the original work is properly cited.

\begin{abstract}
Resistin (RETN) is a gene coding for proinflammatory adipokine called resistin secreted by macrophages in humans. Single nucleotide polymorphisms (SNPs) in RETN are linked to obesity and insulin resistance in various populations. Using dbSNP, 78 nonsynonymous SNPs (nsSNPs) were retrieved and tested on a PredictSNP 1.0 megaserver. Among these, 15 nsSNPs were predicted as highly deleterious and thus subjected to further analyses, such as conservation, posttranscriptional modifications, and stability. The 3D structure of human resistin was generated by homology modeling using Swiss model. Root-mean-square deviation (RMSD), hydrogen bonds (h-bonds), and interactions were estimated. Furthermore, UTRscan served to identify UTR functional SNPs. Among the 15 most deleterious nsSNPs, 13 were predicted to be highly conserved including variants in posttranslational modification sites. Stability analysis predicted 9 nsSNPs (I32S, C51Y, G58E, G58R, C78S, G79C, W98C, $\mathrm{C} 103 \mathrm{G}$, and $\mathrm{C} 104 \mathrm{Y}$ ) which can decrease protein stability with at least three out of the four algorithms used in this study. These nsSNPs were chosen for structural analysis. Both variants C51Y and C104Y showed the highest RMS deviations (1.137 A and $1.308 \AA$, respectively) which were confirmed by the important decrease in total h-bonds. The analysis of hydrophobic and hydrophilic interactions showed important differences between the native protein and the 9 mutants, particularly I32S, G79C, and C104Y. Six SNPs in the 3'UTR (rs920569876, rs74176247, rs1447199134, rs943234785, rs76346269, and rs78048640) were predicted to be implicated in polyadenylation signal. This study revealed 9 highly deleterious SNPs located in the human RETN gene coding region and 6 SNPs within the $3^{\prime}$ UTR that may alter the protein structure. Interestingly, these SNPs are worth to be analyzed in functional studies to further elucidate their effect on metabolic phenotype occurrence.
\end{abstract}

\section{Introduction}

Genomic variation understanding is one of the major challenges of current genomics research field, due to the enormous number of genetic variations in the human genome. Single nucleotide polymorphisms (SNPs) represent the most abundant genetic variations throughout the human genome ranging between 3 and 5 million in each individual [1].
Mostly, SNPs are neutral, but some of them contribute to disease predisposition by modifying protein function or as genetic markers in order to find nearby disease-causing mutations through genetic association studies and familybased studies [2]. Scientists believe that these variants may also influence the response to some drugs [3].

SNPs that change the encoded amino acids are called nonsynonymous single nucleotide polymorphisms (nsSNPs). 
Nonsynonymous SNPs, forming about half of all genetic changes related to human diseases, can influence resulting protein structure and/or function with either neutral or deleterious effects $[4,5]$.

Moreover, the study of noncoding DNA is also important because it contains the majority of reported SNPs in human genome. Polymorphisms in $5^{\prime}$ and $3^{\prime}$ untranslated regions (UTRs) are of major interest because they can affect gene expression and posttranscriptional and posttranslational activities and thus be of functional relevance $[6,7]$.

Resistin is a proinflammatory adipokine which belongs to the cysteine-rich C-terminal domain proteins called resistin-like molecules (RELMs) and mainly secreted by adipocytes in rodents and macrophages in humans $[8,9]$. The gene encoding resistin (RETN) is located on chromosome $19 \mathrm{p} 13.2$. It was shown that resistin is linked to several inflammatory disorders including obesity, type 2 diabetes, cardiovascular disease, and asthma [10-13]. This protein has effects which antagonize insulin action. Some studies have shown that resistin affects glucose transport and causes insulin-stimulated insulin receptor substrate-1 (IRS-1) degradation leading to insulin resistance induction [14-16]. Circulating resistin levels were reported to be significantly increased in both genetically and diet-induced obese mice and decreased with the administration of the antidiabetic drug Rosiglitazone [8].

Moreover, a case-control study on type 1 diabetes mellitus patients showed that the combination of insulin and Rosiglitazone decreased resistin and leptin levels significantly [17]. Genetic variants in RETN showed a significant association with circulating resistin levels. Beckers et al. identified the first missense mutation $\mathrm{C} 78 \mathrm{~S}$ in resistin in a morbidly obese proband and his obese mother. This finding encourages the study of variants in the RETN gene coding region to elucidate their involvement in pathogenesis [18]. It was estimated that genetic factors can explain up to $70 \%$ of the variation in circulating resistin levels [19]. However, analyses of the association between SNPs of the RETN gene and anthropometric variables and alterations related to obesity revealed inconsistent results [10, 20-23].

Basing on the importance of RETN gene in multiple inflammatory diseases, particularly metabolic abnormalities, we conducted a computational analysis using nsSNP effect predictors like SIFT, PolyPhen, PANTHER, PhD-SNP and PredictSNP. Most deleterious nsSNPs were further analyzed by conservation and stability tools. Finally, a structural analysis was conducted in order to identify the most functionally deleterious SNPs in coding and untranslated regions.

\section{Material and Methods}

2.1. Dataset Collection. The SNP information of RETN gene was collected from dbSNP (http://www.ncbi.nlm.nih.gov/ $\mathrm{snp} /$ ). The amino acid sequence of the protein (NCBI accession: NP_001180303) was retrieved from the NCBI protein database (http://www.ncbi.nlm.nih.gov/protein). The theoretical structure of resistin (PDB ID: 1LV6) was abandoned since it was not in agreement with the crystal structure available for mouse resistin now.
2.2. Prediction of Deleterious nsSNPs. PredictSNP1.0 (http:// loschmidt.chemi.muni.cz/predictsnp1/) [24] was used as the predictor of the SNP effect on protein function. This resource is a consensus classifier that enables access to the nine best performing prediction tools: SIFT, PolyPhen-1, PolyPhen-2, MAPP, PhD-SNP, SNAP, PANTHER, PredictSNP, and nsSNPAnalyzer.

SIFT (Sorting Intolerant from Tolerant) predicts whether an amino acid substitution affects the protein function based on sequence homology and the physical properties of amino acids [25]. SIFT takes a query sequence and uses multiple alignment information to predict tolerated and deleterious substitutions in every position of the query sequence. PolyPhen-1 uses expert set of empirical rules to predict possible impact of amino acid substitutions, while PolyPhen-2 (Polymorphism Phenotyping v2) predicts the potential effect of an amino acid substitution on the structure and function of a human protein using multiple sequence alignment and structural information. MAPP (Multivariate Analysis of Protein Polymorphism) analyzes the physicochemical variation present in each column of a protein sequence alignment and predicts the impact of amino acid substitutions on the protein function [26]. PhD-SNP (Predictor of human Deleterious Single Nucleotide Polymorphisms) is a support vector machine- (SVM-) based predictor used to classify nsSNPs into human genetic disease-causing or benign mutations [27]. SNAP (screening for nonacceptable polymorphisms) is a neural network-based method used to predict functional effects of nonsynonymous SNPs using in silico derived protein information [28]. PANTHER (Protein Analysis Through Evolutionary Relationships) estimates the likelihood of a particular nsSNP to cause a functional effect on the protein using position-specific evolutionary preservation [29]. nsSNPAnalyzer uses a machine learning method called random forest to predict whether the nsSNP has a phenotypic effect [30] based on multiple sequence alignment and 3D structure information. Finally, PredictSNP1.0 displays the confidence scores generated by each tool and a consensus prediction as percentages by using their observed accuracy values to simplify comparisons [24].

2.3. Sequence Conservation. A ConSurf web server (http:// consurf.tau.ac.il/) was used to analyze amino acid sequence conservation. This web-based algorithm predicts the crucial functional regions of a protein by estimating the degree of amino acid conservation based on multiple sequence alignment. The grade range from 1 to 9 estimates the extent of conservation of the amino acid throughout evolution. Therefore, grade 9 represents the most highly conserved residue, and the numbers descend to 1 representing the least conserved region. This tool analyzes the conservation at the nucleotide and amino acid levels.

2.4. Prediction of Posttranslational Modification Sites. A ModPred web server (http://www.modpred.org/) was used to predict posttranslational modification (PTM) sites; the server consists of a set of bootstrapped logistic regression models for each type of PTM, retrieved from 126,036 nonredundant PTM sites verified experimentally, the 
literature, and from the databases [31]. Results are given as residue, modification, score, confidence, and remarks. In this study, only medium and high confidence PTMs were taken into consideration.

2.5. Prediction of Change in Protein Stability. The change in protein stability due to nsSNPs was predicted using IMutant2.0 (http://folding.biofold.org/cgi-bin/i-mutant2.0), which is a support vector machine (SVM) web-based tool used for the automatic prediction of changes in protein stability due to SNP. It provides the predicted free energy change value (DDG) and the sign of the prediction as increase or decrease. DDG value is calculated from the unfolding Gibbs free energy value of the mutated protein minus the unfolding Gibbs free energy value of the wild type in $\mathrm{kcal} / \mathrm{mol}$. DDG $>0$ means that the protein stability increased, and $\mathrm{DDG}<0$ means that the protein stability decreased [32].

The stability was also checked by a MUpro tool (http:// mupro.proteomics.ics.uci.edu/). This server is based on two machine learning methods: support vector machines and neural networks. Both of them were trained on a large mutation dataset and showed accuracy above $84 \%$.

This protein calculates a score between -1 and 1 as the confidence of prediction. The confidence score $<0$ indicates that the mutation decreases the protein stability, while a confidence score $>0$ means that the mutation increases the protein stability [33].

2.6. Scanning of UTR SNPs in the UTR Site. The $5^{\prime}$ and $3^{\prime}$ untranslated regions (UTRs) have crucial roles in degradation, translation, and localization of mRNAs as well as the regulation of protein-protein interaction. We used the UTRscan web server http://itbtools.ba.itb.cnr.it/utrscan to predict the functional SNPs in the $5^{\prime}$ and $3^{\prime}$ UTRs. The UTRscan tool allows the enquirer to search user-submitted sequences for any of the motifs present in UTRsite. UTRsite derives data from UTRdb, a curated database that updates UTR datasets through primary data mining and experimental validation $[7,34]$. To perform this analysis, the primary FASTA format data was submitted and the results were showed in the form of signal names and their positions in the transcript.

\subsection{Structural Analysis}

2.7.1. Modeling of Native and Mutant Structure. The transcript with the reference sequence NP_001180303.1 was used for the homology modeling. We selected the X-ray crystal structure of Mus musculus resistin from the Protein Data Bank (PDB) with PDB code 1RGX [9] as a template to generate a human resistin by homology modeling using the Swiss model platform (https://swissmodel.expasy.org). The model has a QMEAN of -1.83 and a sequence identity of $55.56 \%$ (Figure 1).

UCSF Chimera was used to confirm the corresponding positions of the SNPs and to construct the 15 mutant models [35]. It is a highly extensible program developed by the Resource for Biocomputing, Visualization, and Informatics at the University of California, San Francisco, for

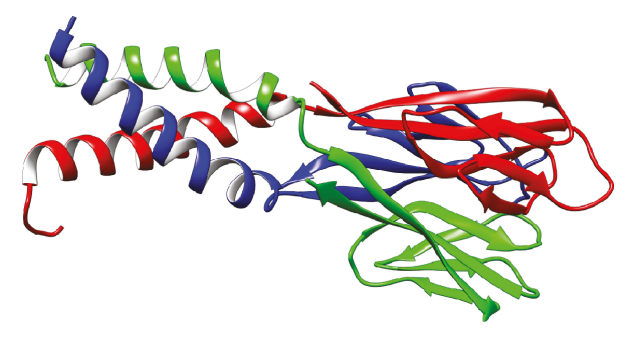

FIgURE 1: The tridimensional model of human resistin generated using homology modeling by a Swiss model web server. The model is a trimer with three chains A (red), B (green), and C (blue).

interactive visualization and analysis of molecular structures and related data.

The energy minimization of the wild type and mutant structures was performed by NOMAD-Ref server Gromacsbased as a default force field; we used conjugate gradient method for the 3D structure optimization [36].

2.7.2. RMSD and Total Hydrogen Bond Prediction. UCSF Chimera served again to check RMS deviation by superimposing both native and mutant structures. In addition, this tool served to calculate total h-bond values for each structure.

2.7.3. Interaction Analysis. COCOMAPS (bioCOmplexes COntact MAPS) is a web application to effectively analyze and visualize the interface in biological protein-protein complexes by making use of intermolecular contact maps. The input file was the resistin homology model in PDB format. In our study, we used COCOMAPS to analyze the interaction between the three monomers of resistin protein [37]. To achieve this, we uploaded the PDB file of resistin trimer ( $\mathrm{A}, \mathrm{B}$, and $\mathrm{C}$ as chain IDs for each monomer) and we then compared the interaction interfaces between the two chains A and B considered as Molecule 1 interacting with the third chain $\mathrm{C}$ considered as Molecule 2 (interactions include residues from chain $\mathrm{A}$ and from chain $\mathrm{B}$ together interacting with chain $\mathrm{C}$ ).

2.7.4. Prediction of Protein-Protein Interactions. STRING (Search Tool for the Retrieval of Interacting Genes/Proteins, available at http://string-db.org) is a database of known and predicted protein interactions, which currently covers $9,643,763$ proteins from 2031 organisms. This database provides a critical assessment and integration of protein-protein interactions including direct (physical) and indirect (functional) associations [38].

\section{Results}

3.1. SNP Datasets. The RETN SNP data investigated in this work was retrieved in early October 2018 from dbSNP database (http://www.ncbi.nlm.nih.gov/snp/?term=RETN). It contained a total of 1075 SNPs. Out of which, 78 were nsSNPs, 35 were coding synonymous SNPs, 339 were located in the noncoding region, which comprises 18 SNPs in the $5^{\prime} \mathrm{UTR}, 35$ SNPs were in the $3^{\prime} \mathrm{UTR}$, and 287 were in the intronic region. 
TABLE 1: The expected accuracy results of the SNPs of RETN predicted as deleterious in PredictSNP and integrated tools.

\begin{tabular}{lcccccccc}
\hline nsSNPs & ID variants & PredictSNP & PolyPhen-1 & PolyPhen-2 & SIFT & MAPP & PhD-SNP & SNAP \\
\hline I32S & rs894321927 & $(0.86)$ & $(0.74)$ & $(0.60)$ & $(0.79)$ & $(0.77)$ & $(0.85)$ & $(0.72)$ \\
C51Y & rs759129635 & $(0.86)$ & $(0.74)$ & $(0.81)$ & $(0.79)$ & $(0.77)$ & $(0.81)$ & $(0.86)$ \\
G58R & rs760260537 & $(0.86)$ & $(0.74)$ & $(0.81)$ & $(0.79)$ & $(0.87)$ & $(0.73)$ & $(0.88)$ \\
G58E & rs763916942 & $(0.86)$ & $(0.74)$ & $(0.81)$ & $(0.79)$ & $(0.91)$ & $(0.58)$ & $(0.88)$ \\
G66R & rs566843624 & $(0.86)$ & $(0.74)$ & $(0.56)$ & $(0.42)$ & $(0.62)$ & $(0.81)$ & $(0.86)$ \\
G71R & rs772946179 & $(0.86)$ & $(0.74)$ & $(0.59)$ & $(0.79)$ & $(0.87)$ & $(0.57)$ & $(0.86)$ \\
C74W & rs532089804) & $(0.86)$ & $(0.74)$ & $(0.81)$ & $(0.79)$ & $(0.91)$ & $(0.77)$ & $(0.75)$ \\
C78S & rs199834487 & $(0.86)$ & $(0.74)$ & $(0.81)$ & $(0.79)$ & $(0.75)$ & $(0.67)$ & $(0.86)$ \\
G79C & rs111331676 & $(0.86)$ & $(0.74)$ & $(0.81)$ & $(0.79)$ & $(0.85)$ & $(0.73)$ & $(0.86)$ \\
R84C & rs779335092 & $(0.86)$ & $(0.74)$ & $(0.81)$ & $(0.79)$ & $(0.85)$ & $(0.73)$ & $(0.84)$ \\
D97E & rs768223197 & $(0.86)$ & $(0.59)$ & $(0.81)$ & $(0.79)$ & $(0.77)$ & $(0.58)$ & $(0.84)$ \\
W98C & rs776285077 & $(0.86)$ & $(0.74)$ & $(0.81)$ & $(0.79)$ & $(0.85)$ & $(0.81)$ & $(0.86)$ \\
W98L & rs1035187378 & $(0.86)$ & $(0.74)$ & $(0.81)$ & $(0.52)$ & $(0.91)$ & $(0.58)$ & $(0.88)$ \\
C103G & rs991039386 & $(0.86)$ & $(0.74)$ & $(0.81)$ & $(0.79)$ & $(0.76)$ & $(0.58)$ & $(0.88)$ \\
C104Y & rs891939673 & $(0.86)$ & $(0.74)$ & $(0.81)$ & $(0.79)$ & $(0.77)$ & $(0.81)$ & $(0.88)$ \\
\hline
\end{tabular}

3.2. Prediction of Deleterious nsSNPs. A total of $78 \mathrm{nsSNPs}$ were selected for our investigation. This SNP collection was analyzed with various in silico prediction tools to measure their effects on pathogenicity and to find out diseaseassociated SNPs. All nsSNPs which were obtained from SNP database were loaded to PredictSNP1.0, and all available integrated tools were selected for prediction. Fifteen nsSNPs were predicted as deleterious by all integrated tools, except for nsSNPAnalyzer and PANTHER that did not give any prediction for any mutation. According to SNAP, a total of 38 nsSNPs out of 54 were predicted to be deleterious $(70.37 \%)$, followed by MAPP with 37 deleterious nsSNPs (68.51\%), PolyPhen-2 with 31 nsSNPs (57.40\%), PolyPhen-1 with 25 nsSNPs (46.29\%), SIFT with 26 nsSNPs (48.15\%), and PhD-SNP with 18 nsSNPs (33.33\%). The nsSNPs predicted as deleterious are listed in Table 1 with the expected accuracy and are selected for further analysis (Table 1).

3.3. Analysis of Conservation. The results of ConSurf analysis showed that 13 deleterious missense SNPs are located in highly conserved regions, with conservation values ranging between 7 and 9, which suggests that these positions are important for the resistin integrity. Among these, three residues were predicted to be exposed and functional, five others were predicted to be buried and structural, two buried residues and one exposed residue." while we should mention at the beginning of the paragraph that "11 deleterious missence SNPs are located in highly conserved regions", because we mentionned just after this that conservation values are ranging between 7 and 9 so we excluded G71 ( score: 4) and R84 (score: 6). The position 84 was predicted as moderately conserved, and the position 71 was predicted as variable residue; therefore, they were not selected for structural analysis.

3.4. Prediction of Posttranslational Modification Sites. ModPred was used to predict posttranslational modification sites present within the human resistin protein. Only
PTMs with high or medium confidence were discussed. In the native protein, position R84 was predicted as a site of ADP-ribosylation, W98 as a site of C-linked glycosylation or proteolytic cleavage, and C103 and C104 as disulfide linkage sites. After mutagenesis, C51 appeared as a site of amidation with the change of Cys to Tyr, while the position W98 changed to a disulfide linkage site with the change of Trp to Cys. Regarding the position C104, it was predicted that the change of Cys to Tyr conferred an amidation site with a high confidence. The results of ModPred are shown in Table 2.

3.5. The Impact of Predicted Deleterious Mutations on Resistin Protein Stability. We analyzed the 13 missense substitutions predicted as deleterious from the previous steps with the I-Mutant2.0. and MUpro web server. nsSNPs predicted to decrease stability with both tools were selected for further structural analysis. The results are showed in Table 3 .

\subsection{Structural Analysis}

3.6.1. Modeling of Human Resistin Structure. Using the X-ray crystal structure (1rgx) as a template, we modeled the 3D structure of native human resistin using the Swiss model web server. Figure 2 showed the generated model as a trimer with three monomers (A, B, and $\mathrm{C}$ ). This trimer was used to construct the 9 mutant models of human resistin.

3.6.2. RMSD Difference and Total Hydrogen Bonds. The RMSD values associated with the 9 mutants are given in Table 4. As the RMSD value increases, the deviation between native- and mutant-type structures will be higher and thus may induce a change in protein activity. Altered C51Y and C104Y mutants showed the highest RMSD; results are shown in Figures 2(a) and 2(b). In addition, total h-bonds were calculated to assess their contribution in the stability and the folding of the native protein. All 
TABLE 2: Details of nsSNPs selected as deleterious among the reported SNPs, their conservation analysis by ConSurf, and posttranslational modification site prediction by ModPred.

\begin{tabular}{|c|c|c|c|c|c|c|}
\hline Position & Conservation score & $\mathrm{B} / \mathrm{E}$ & $\mathrm{F} / \mathrm{S}$ & PTM sites (wild type) & Variants & PTM sites (mutants) \\
\hline $\mathrm{I} 32$ & 7 & $\mathrm{~B}$ & - & - & I32S & - \\
\hline C51 & 9 & $\mathrm{~B}$ & S & - & C51Y & $\begin{array}{c}\text { Amidation } \\
\text { Phosphorylation }\end{array}$ \\
\hline \multirow{2}{*}{ G58 } & \multirow{2}{*}{9} & \multirow{2}{*}{$\mathrm{E}$} & \multirow{2}{*}{$\mathrm{F}$} & \multirow{2}{*}{ Proteolytic cleavage } & G58R & Proteolytic cleavage \\
\hline & & & & & G58E & Proteolytic cleavage \\
\hline G66 & 7 & $\mathrm{E}$ & - & Proteolytic cleavage & G66R & Proteolytic cleavage \\
\hline G71 & 4 & B & - & - & G71R & $\begin{array}{l}\text { Proteolytic cleavage } \\
\text { ADP-ribosylation }\end{array}$ \\
\hline $\mathrm{C} 74$ & 9 & B & $S$ & Disulfide linkage & C74W & - \\
\hline $\mathrm{C} 78$ & 9 & B & S & Disulfide linkage & C78S & O-linked glycosylation \\
\hline G79 & 9 & $\mathrm{E}$ & $\mathrm{F}$ & - & G79C & Disulfide linkage \\
\hline R84 & 6 & $\mathrm{E}$ & - & $\begin{array}{l}\text { ADP-ribosylation } \\
\text { Proteolytic cleavage }\end{array}$ & $\mathrm{R} 84 \mathrm{C}$ & Disulfide linkage \\
\hline D97 & 9 & $\mathrm{E}$ & $\mathrm{F}$ & - & D97E & Disulfide linkage \\
\hline \multirow[b]{2}{*}{ W98 } & \multirow[b]{2}{*}{8} & \multirow[b]{2}{*}{ B } & \multirow[b]{2}{*}{-} & C-linked glycosylation & W98C & Disulfide linkage \\
\hline & & & & $\begin{array}{c}\text { Proteolytic cleavage } \\
\text { Amidation }\end{array}$ & W98L & - \\
\hline C103 & 9 & B & $S$ & Disulfide linkage & C103 & Proteolytic cleavage \\
\hline C104 & 9 & B & $S$ & Disulfide linkage & $\mathrm{C} 104$ & $\begin{array}{c}\text { Amidation } \\
\text { Proteolytic cleavage }\end{array}$ \\
\hline
\end{tabular}

PTM: posttranslational modification; B: buried; E: exposed; F: functional; S: structural.

Table 3: Prediction of change in protein stability using I-Mutant2.0 and MUpro.

\begin{tabular}{|c|c|c|c|c|}
\hline \multirow{2}{*}{ Position } & \multirow{2}{*}{$\begin{array}{c}\text { I-Mutant } 2.0 \\
\text { DDG value }(\mathrm{kcal} / \mathrm{mol})\end{array}$} & \multicolumn{3}{|c|}{ MUpro } \\
\hline & & DDG value & SVM & $\mathrm{NN}(\mathrm{kcal} / \mathrm{mol})$ \\
\hline $\mathrm{I} 32 \mathrm{~S}$ & -2.45 (decrease) & -1.490 (decrease) & -0.80 (decrease) & -0.96 (decrease) \\
\hline $\mathrm{C} 51 \mathrm{Y}$ & -0.89 (decrease) & -0.234 (decrease) & -0.20 (decrease) & -0.69 (decrease) \\
\hline G58R & -0.15 (decrease) & -0.95 (decrease) & -0.22 (decrease) & -0.73 (decrease) \\
\hline G58E & -1.43 (decrease) & -0.913 (decrease) & 0.23 (increase) & -0.67 (decrease) \\
\hline G66R & -1.74 (decrease) & -0.84 (decrease) & 0.48 (increase) & 0.51 (increase) \\
\hline $\mathrm{C} 74 \mathrm{~W}$ & -0.76 (decrease) & -0.69 (decrease) & 0.54 (increase) & 0.85 (increase) \\
\hline $\mathrm{C} 78 \mathrm{~S}$ & -0.63 (decrease) & -1.513 (decrease) & -0.09 (decrease) & 0.87 (increase) \\
\hline G79C & -0.89 (decrease) & -0.842 (decrease) & -0.84 (decrease) & -0.38 (decrease) \\
\hline D97E & 0.52 (increase) & -0.77 (decrease) & 0.025 (increase) & -0.65 (decrease) \\
\hline W98C & -1.14 (decrease) & -0.442 (decrease) & -0.77 (decrease) & -0.92 (decrease) \\
\hline W98L & -0.59 (decrease) & 0.16 (increase) & -0.10 (decrease) & 0.691 (increase) \\
\hline C103G & -1.05 (decrease) & -1.63 (decrease) & -0.80 (decrease) & 0.547 (increase) \\
\hline C104Y & 0.32 (increase) & -1.03 (decrease) & -0.36 (decrease) & -0.80 (decrease) \\
\hline
\end{tabular}

DDG: delta delta G; SVM: support vector machine; NN: neural network.

mutated structures revealed a change in total h-bonds compared to the native resistin, but the C104Y mutant showed a remarkable decrease forming $254 \mathrm{~h}$-bonds while the native structure formed 291. Moreover, the visualization of native structure showed that $\mathrm{C} 51$ and $\mathrm{C} 104$ residues form a disulfide bond with each other (Figure 2(d)); the change of cysteine carried on the alpha helix in these positions induces the breakage of the disulfide bridge (Figures 2(c) and 2(e)) which may disturb the protein structure.
3.6.3. Interaction Analysis. The interface contacts between the amino acids present within the resistin trimer were studied using COCOMAPS. Variation in the number of different types of interactions was observed between the native and 9 resistin mutants; the results are given in Table 5 .

Regarding the number of hydrophilic-hydrophilic interactions, the native complex participated with 262 hydrophilichydrophilic interactions. The mutant complexes I32S, C51Y, G79C, and C104Y, showed a significant increase in the 


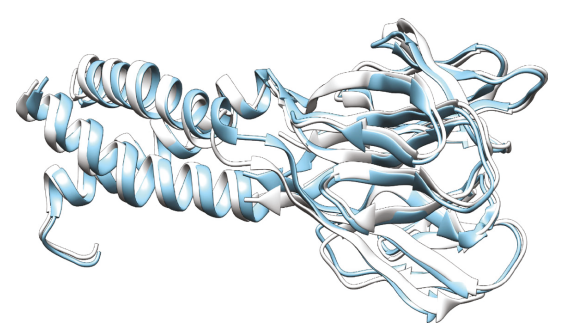

(a)

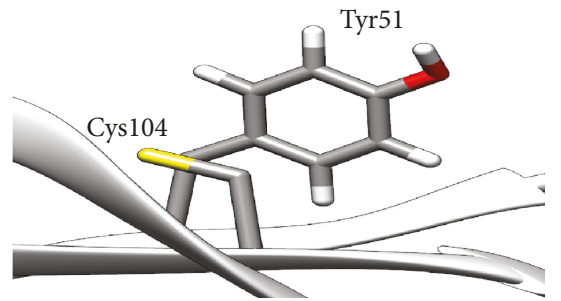

(c)

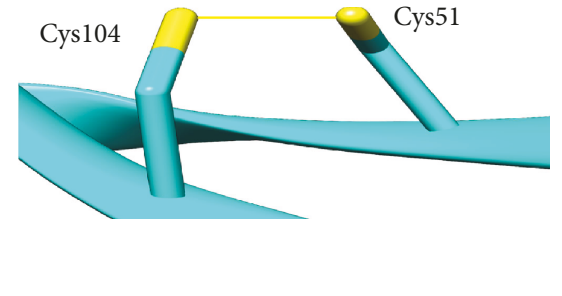

(d)

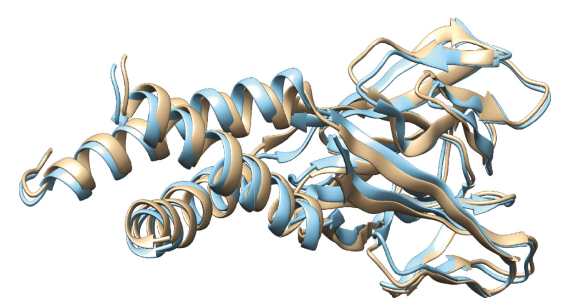

(b)

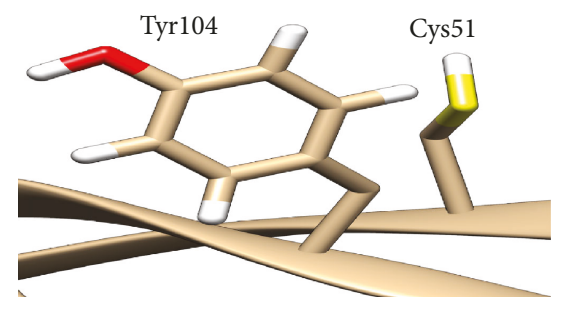

(e)

FIGURE 2: (a) Superimposed of native structure of human resistin (cyan color) onto mutant C51Y structure (white color) showing a deviation RMSD of $1.137 \AA$. (b) Superimposed of native structure of human resistin (cyan color) onto mutant C104Y structure (white color) showing a deviation RMSD of 1.308 A. (c) Disulfide bond breakage in C51Y resistin mutant. (d) Disulfide bond between both cysteine residues 51 and 104 in wild-type resistin. (e) Disulfide bond breakage in C104Y resistin mutant.

TABLE 4: RMSD value and total hydrogen bonds after minimization of each model.

\begin{tabular}{lcc}
\hline & RMSD $(\AA)$ & Total h-bonds \\
\hline Native & 0 & 291 \\
I32S & 0.023 & 297 \\
C51Y & 1.137 & 272 \\
G58E & 0.30 & 297 \\
C78S & 0.37 & 299 \\
G58R & 0.182 & 292 \\
G79C & 0.265 & 296 \\
W98C & 0.158 & 296 \\
C103G & 0.486 & 250 \\
C104Y & 1.308 & 254 \\
\hline
\end{tabular}

RMSD: root-mean-square deviation.

number of hydrophilic-hydrophilic interactions with 286, 266,277 , and 266 interactions, respectively, which indicate a reduction in the hydrophobicity of these mutant trimers. In addition, the mutant complex C103G showed a significant increase in the number of hydrophobic-hydrophobic interactions indicating the increase of its hydrophobicity.

Moreover, we found that the C51Y mutant trimer interacts with only 75 residues of chain $\mathrm{C}$ forming the trimer complex while in the native complex, chain $\mathrm{C}$ interacts with 78 residues. This small deviation may disrupt resistin trimer formation.

3.6.4. Prediction of the Effect of SNPs Located in the UTR by a UTRscan Server. The UTRscan server was used to predict the effect of UTR SNPs on transcriptional motif. Six SNPs in the 3'UTR, namely, rs920569876, rs74176247, rs1447199134, rs943234785, rs76346269, and rs78048640, were predicted to be in polyadenylation sites and thus may be responsible for pathological phenotypes. Results are given in Table 6.

Protein-Protein Interactions Using STRING. Prediction of protein-protein interactions indicated that resistin interacts with 10 proteins including insulin (INS), insulin receptor (INSR), leptin (LEP), adiponectin (ADIPOQ), peroxisome proliferator-activated receptor gamma (PPARG), tumor necrosis factor (TNF), interleukin 6 (TNF), nicotinamide phosphoribosyltransferase (NAMPT), nuclear receptor subfamily 3 group C member 1 (NR3C1), and ghrelin/obestatin prepropeptide (GHRL) (Figure 3).

\section{Discussion}

With the growth of SNP number in databases, it becomes difficult to determine SNPs contributing in disease development. Thus, computational analysis can help to select a limited number of prioritizing deleterious SNPs for genetic disease screening [39]. Among the mutations affecting the protein function, nsSNPs are very frequently occurring in many inherited diseases [40]. Nonsynonymous SNPs were essentially described to inhibit protein activity, DNA-protein, or DNA-miRNA binding. There were many metabolic abnormalities related genes studied with computational approaches in order to predict functional SNPs such as hepatocyte nuclear factor 1 alpha (HNF1A), apolipoprotein AI (APOAI), apolipoprotein E3 (APOE3), and high-density lipoprotein (HDL) [39, 41-44]. To date, more than 70 missense mutations have been reported in human RETN gene. However, population-based association studies on RETN remain insufficient. Therefore, the current study is aimed at investigating the structural and functional impact of the SNPs present in the RETN gene using a thorough 
TABLE 5: Interactions observed between the three monomeric of resistin protein in native and 9 mutant complexes.

\begin{tabular}{lcccccccccc}
\hline & Native & I32S & C51Y & G58E & G58R & C78S & G79C & W98C & C103G & C104Y \\
\hline $\begin{array}{l}\text { Number of interacting residues Molecule 1 } \\
\text { (chain A and chain B) }\end{array}$ & 129 & 129 & 130 & 127 & 129 & 129 & 128 & 130 & 130 & 129 \\
Number of interacting residues Molecule 2 (chain C) & 78 & 78 & 75 & 77 & 78 & 78 & 78 & 78 & 78 & 75 \\
Number of hydrophilic-hydrophobic interaction & 343 & 357 & 329 & 342 & 338 & 334 & 323 & 343 & 357 & 320 \\
Number of hydrophilic-hydrophilic interaction & 262 & 286 & 266 & 257 & 258 & 261 & 277 & 251 & 259 & 266 \\
Number of hydrophobic-hydrophobic interaction & 193 & 175 & 188 & 194 & 196 & 190 & 179 & 194 & 201 & 189 \\
\hline
\end{tabular}

TABLE 6: SNPs (UTR mRNA) that were predicted to be functionally significant by UTRscan.

\begin{tabular}{lccc}
\hline SNP ID & $\begin{array}{c}\text { Nucleotide } \\
\text { change }\end{array}$ & $\begin{array}{c}\text { UTR } \\
\text { position }\end{array}$ & $\begin{array}{c}\text { Functional element } \\
\text { change }\end{array}$ \\
\hline rs920569876 & $\mathrm{A} / \mathrm{G}$ & $3^{\prime} \mathrm{UTR}$ & Polyadenylation signal \\
rs74176247 & $\mathrm{A} / \mathrm{G}$ & $3^{\prime} \mathrm{UTR}$ & Polyadenylation signal \\
rs1447199134 & $\mathrm{A} / \mathrm{T}$ & $3^{\prime} \mathrm{UTR}$ & Polyadenylation signal \\
rs943234785 & $\mathrm{A} / \mathrm{G}$ & $3^{\prime} \mathrm{UTR}$ & Polyadenylation signal \\
rs76346269 & $\mathrm{A} / \mathrm{T}$ & $3^{\prime} \mathrm{UTR}$ & Polyadenylation signal \\
rs78048640 & $\mathrm{A} / \mathrm{G}$ & $3^{\prime} \mathrm{UTR}$ & Polyadenylation signal \\
\hline
\end{tabular}

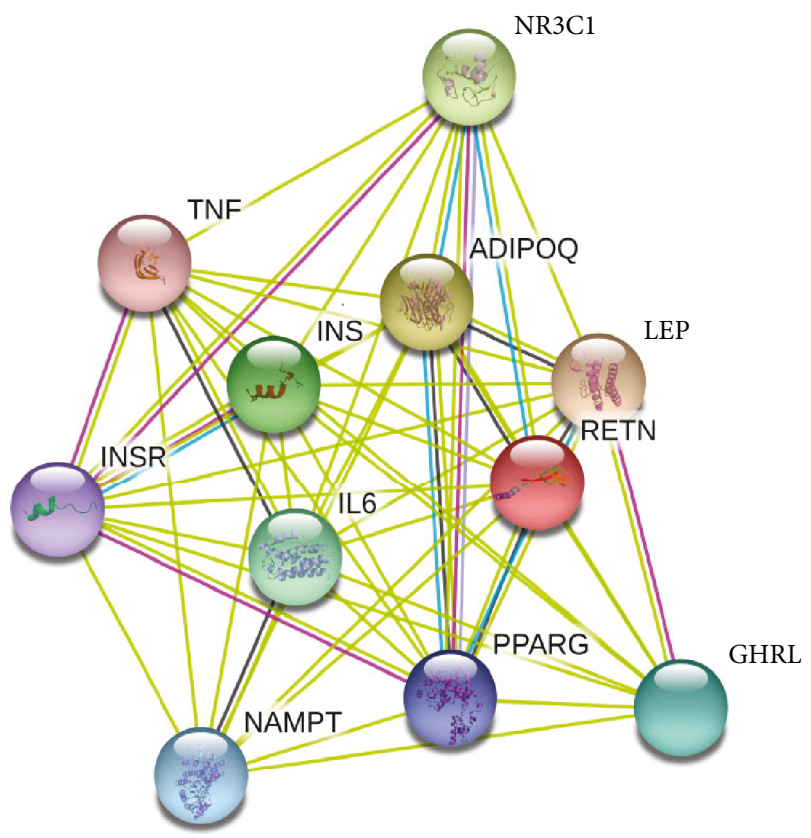

FIGURE 3: Protein-protein interaction network of resistin using a STRING server.

computational analysis. A total of $78 \mathrm{nsSNPs}$ in RETN gene were retrieved from dbSNP database.

The methods used in this study revealed the importance of using various algorithms with different prediction capacities to estimate the effect of variations on structural and functional levels. Using a single bioinformatic tool to predict potentially pathogenic nsSNPs may not be significant [45]. Hence, the present study was based on multiple computational tools including SIFT, Poly-Phen1/2, MAAP, PhD-
SNP, PredictSNP, SNAP, ConSurf, ModPred, I-Mutant2.0, and MUpro in order to identify the most deleterious nsSNPs in RETN gene.

Fifteen nsSNPs were predicted to be the most deleterious SNPs by these tools. In addition, using the ConSurf web server, 13 of them were predicted to be highly conserved with conservation scores ranging between 7 and 9. Moreover, PTM site prediction showed that W98 is a site for ADPribosylation and proteolytic cleavage in the wild type, while after mutation (W98C), it became a site for disulfide linkage with the gain of Cys residue; this may induce conformational restrictions on the protein, altering strongly its folding, function, and stability. Inversely, C103 and C104 residues were predicted as a PTM sites that lost disulfide linkages. Although disulfide bond 3D structure and interactions are highly conserved in nature, their loss may affect the protein folding. Nine nsSNPs were significantly predicted to affect stability. These results suggested that I32S, C51Y, G58E, G58R, C78S, G79C, W98C, C103G, and C104Y may be the structurally and functionally most significant SNPs in human resistin. The variant $\mathrm{C} 104 \mathrm{Y}$ possessed the highest RMSD value, i.e., $1.308 \AA$ followed by C51Y with an RMSD of $1.137 \AA$. Moreover, these variants had a significant loss of hydrogen bonds compared to the wild type. Interestingly, C51, C78, C103, and C104 residues are located in the highly conserved cysteine-rich C-terminus of resistin as all proteins of the resistin-like family share a common $\mathrm{C}$-terminus domain with invariant spacing between cysteine positions (1C-X11-2C-X8-3C-X-4C-X3-5C-X10-6C-X7C-X-8C-X9-9C-10C) [8, 46, 47]. Figures 2(c) and 2(e) showed C51Y and C104Y mutants that lost the disulfide bridge after a change of cysteine to tyrosine residues. This could be expected to destabilize the helix carrying residues that may be involved in binding sites with other proteins. Using STRING, the functional network of resistin interactions with ten different proteins implicated unanimously in inflammatory and metabolic pathways suggests the strong implication of resistin in metabolic abnormalities.

\section{Conclusion}

RETN gene was investigated in this work by assessing the impact of deleterious SNPs in coding and untranslated regions through a computational approach. In a total of 78 missense SNPs, 15 were predicted as the most deleterious using PredictSNP. From which, 9 nsSNPs were predicted as highly conserved and affect protein stability. The structural analysis revealed high RMSD scores for both C104Y and 
C51Y variants, respectively, with a loss of the total hydrogen bonds. Six UTR SNPs were predicted to be in polyadenylation sites. Hence, we concluded that 9 nsSNPs I32S, C51Y, G58E, G58R, C78S, G79C, W98C, C103G, and C104Y and 6 substitutions in the $3^{\prime}$ UTR, namely, rs920569876, rs74176247, rs1447199134, rs943234785, rs76346269, and rs78048640, could be important candidates in the pathological process of resistin particularly in metabolic pathways.

\section{Data Availability}

All data generated or analysed during this study are included in this published article.

\section{Conflicts of Interest}

Authors declare that they have no conflict of interest.

\section{Authors' Contributions}

Lamiae Elkhattabi and Imane Morjane have contributed equally to the work.

\section{Acknowledgments}

The authors are thankful to Pasteur Institute of Morocco for providing encouragement and facilities.

\section{References}

[1] T. C. Dakal, D. Kala, G. Dhiman, V. Yadav, A. Krokhotin, and N. V. Dokholyan, "Predicting the functional consequences of non-synonymous single nucleotide polymorphisms in IL8 gene," Scientific Reports, vol. 7, no. 1, p. 6525, 2017.

[2] X. Wang, D. Tomso, X. Liu, and D. Bell, "Single nucleotide polymorphism in transcriptional regulatory regions and expression of environmentally responsive genes," Toxicology and Applied Pharmacology, vol. 207, no. 2, pp. 84-90, 2005.

[3] K. M. Giacomini, C. M. Brett, R. B. Altman et al., "The pharmacogenetics research network: from SNP discovery to clinical drug response," Clinical Pharmacology \& Therapeutics, vol. 81, no. 3, pp. 328-345, 2007.

[4] M. Cargill, D. Altshuler, J. Ireland et al., "Characterization of single-nucleotide polymorphisms in coding regions of human genes," Nature Genetics, vol. 22, no. 3, pp. 231-238, 1999.

[5] P. Yue and J. Moult, "Identification and analysis of deleterious human SNPs," Journal of Molecular Biology, vol. 356, no. 5, pp. 1263-1274, 2006.

[6] C. Mayr, "Regulation by 3 '-untranslated regions," Annual Review of Genetics, vol. 51, no. 1, pp. 171-194, 2017.

[7] S. Chatterjee and J. K. Pal, "Role of $5^{\prime}$ - and $3^{\prime}$-untranslated regions of mRNAs in human diseases," Biology of the Cell, vol. 101, no. 5, pp. 251-262, 2009.

[8] C. M. Steppan, S. T. Bailey, S. Bhat et al., "The hormone resistin links obesity to diabetes," Nature, vol. 409, no. 6818, pp. 307-312, 2001.

[9] S. D. Patel, L. Rossetti, P. E. Scherer, and L. Shapiro, "Disulfide-dependent multimeric assembly of resistin family hormones," Science, vol. 304, no. 5674, pp. 1154-1158, 2004.
[10] J. C. Engert, M.-C. Vohl, S. M. Williams et al., “5' flanking variants of resistin are associated with obesity," Diabetes, vol. 51, no. 5, pp. 1629-1634, 2002.

[11] N. Rathwa, R. Patel, S. P. Palit, A. V. Ramachandran, and R. Begum, "Genetic variants of resistin and its plasma levels: association with obesity and dyslipidemia related to type 2 diabetes susceptibility," Genomics, 2018.

[12] D. Ziora, E. Machura, K. T. Ziora, E. Swietochowska, J. M. Oswiecimska, and A. Kasperska-Zajac, "Serum resistin levels are elevated in schoolchildren with atopic asthma," Neuro Endocrinology Letters, vol. 34, no. 3, pp. 212-216, 2013.

[13] S. Demirci, A. Aynalı, K. Demirci, S. Demirci, and B. C. Arıdoğan, "The serum levels of resistin and its relationship with other proinflammatory cytokines in patients with Alzheimer's disease," Clinical Psychopharmacology and Neuroscience, vol. 15, no. 1, pp. 59-63, 2017.

[14] C. M. Steppan, J. Wang, E. L. Whiteman, M. J. Birnbaum, and M. A. Lazar, "Activation of SOCS-3 by resistin," Molecular and Cellular Biology, vol. 25, no. 4, pp. 1569-1575, 2005.

[15] A. J. Gerrits, E. Gitz, C. A. Koekman, F. L. Visseren, T. W. van Haeften, and J. W. N. Akkerman, "Induction of insulin resistance by the adipokines resistin, leptin, plasminogen activator inhibitor- 1 and retinol binding protein 4 in human megakaryocytes," Haematologica, vol. 97, no. 8, pp. 1149$1157,2012$.

[16] L. Rui, M. Yuan, D. Frantz, S. Shoelson, and M. F. White, "SOCS-1 and SOCS-3 block insulin signaling by ubiquitinmediated degradation of IRS1 and IRS2," Journal of Biological Chemistry, vol. 277, no. 44, pp. 42394-42398, 2002.

[17] M. Guclu, O. Oz Gul, S. Cander et al., "Effect of rosiglitazone and insulin combination therapy on inflammation parameters and adipocytokine levels in patients with type $1 \mathrm{DM}$," Journal of Diabetes Research, vol. 2015, Article ID 807891, 9 pages, 2015.

[18] S. Beckers, F. de Freitas, D. Zegers et al., "Identification and functional characterization of a missense mutation in resistin in two patients with severe obesity and insulin resistance," European Journal of Endocrinology, vol. 164, no. 6, pp. 927936, 2011.

[19] C. Menzaghi, A. Coco, L. Salvemini et al., "Heritability of serum resistin and its genetic correlation with insulin resistance-related features in nondiabetic Caucasians," The Journal of Clinical Endocrinology \& Metabolism, vol. 91, no. 7, pp. 2792-2795, 2006.

[20] G. D. Norata, M. Ongari, K. Garlaschelli et al., "Effect of the $-420 \mathrm{C} / \mathrm{G}$ variant of the resistin gene promoter on metabolic syndrome, obesity, myocardial infarction and kidney dysfunction," Journal of Internal Medicine, vol. 262, no. 1, pp. 104112, 2007.

[21] L. Bouchard, S. J. Weisnagel, J. C. Engert et al., "Human resistin gene polymorphism is associated with visceral obesity and fasting and oral glucose stimulated C-peptide in the Québec Family Study," Journal of Endocrinological Investigation, vol. 27, no. 11, pp. 1003-1009, 2004.

[22] Y. M. Cho, B.-S. Youn, S. S. Chung et al., "Common genetic polymorphisms in the promoter of resistin gene are major determinants of plasma resistin concentrations in humans," Diabetologia, vol. 47, no. 3, pp. 559-565, 2004.

[23] V. S. Mattevi, V. M. Zembrzuski, and M. H. Hutz, "A resistin gene polymorphism is associated with body mass index in women," Human Genetics, vol. 115, no. 3, pp. 208-212, 2004. 
[24] J. Bendl, J. Stourac, O. Salanda et al., "PredictSNP: robust and accurate consensus classifier for prediction of disease-related mutations," PLoS Computational Biology, vol. 10, no. 1, article e1003440, 2014

[25] P. C. Ng and S. Henikoff, "SIFT: predicting amino acid changes that affect protein function," Nucleic Acids Research, vol. 31, no. 13, pp. 3812-3814, 2003.

[26] E. A. Stone and A. Sidow, "Physicochemical constraint violation by missense substitutions mediates impairment of protein function and disease severity," Genome Research, vol. 15, no. 7, pp. 978-986, 2005.

[27] E. Capriotti, R. Calabrese, and R. Casadio, "Predicting the insurgence of human genetic diseases associated to single point protein mutations with support vector machines and evolutionary information," Bioinformatics, vol. 22, no. 22, pp. 2729-2734, 2006.

[28] Y. Bromberg and B. Rost, "SNAP: predict effect of nonsynonymous polymorphisms on function," Nucleic Acids Research, vol. 35, no. 11, pp. 3823-3835, 2007.

[29] H. Tang and P. D. Thomas, "PANTHER-PSEP: predicting disease-causing genetic variants using position-specific evolutionary preservation," Bioinformatics, vol. 32, no. 14, pp. 2230-2232, 2016.

[30] L. Bao, M. Zhou, and Y. Cui, "nsSNPAnalyzer: identifying disease-associated nonsynonymous single nucleotide polymorphisms," Nucleic Acids Research, vol. 33, Supplement 2, pp. W480-W482, 2005.

[31] V. Pejaver, W.-L. Hsu, F. Xin, A. K. Dunker, V. N. Uversky, and P. Radivojac, "The structural and functional signatures of proteins that undergo multiple events of post-translational modification," Protein Science, vol. 23, no. 8, pp. 1077-1093, 2014.

[32] E. Capriotti, P. Fariselli, and R. Casadio, "I-Mutant2.0: predicting stability changes upon mutation from the protein sequence or structure," Nucleic Acids Research, vol. 33, Supplement 2, pp. W306-W310, 2005.

[33] J. Cheng, A. Randall, and P. Baldi, "Prediction of protein stability changes for single-site mutations using support vector machines," Proteins, vol. 62, no. 4, pp. 1125-1132, 2006.

[34] G. Pesole, S. Liuni, G. Grillo et al., "UTRdb and UTRsite: specialized databases of sequences and functional elements of $5^{\prime}$ and $3^{\prime}$ untranslated regions of eukaryotic mRNAs. Update 2002," Nucleic Acids Research, vol. 30, no. 1, pp. 335-340, 2002.

[35] E. F. Pettersen, T. D. Goddard, C. C. Huang et al., "UCSF Chimera-a visualization system for exploratory research and analysis," Journal of Computational Chemistry, vol. 25, no. 13, pp. 1605-1612, 2004.

[36] E. Lindahl, C. Azuara, P. Koehl, and M. Delarue, "NOMADRef: visualization, deformation and refinement of macromolecular structures based on all-atom normal mode analysis," Nucleic Acids Research, vol. 34, Supplement 2, pp. W52W56, 2006.

[37] A. Vangone, R. Spinelli, V. Scarano, L. Cavallo, and R. Oliva, "COCOMAPS: a web application to analyze and visualize contacts at the interface of biomolecular complexes," Bioinformatics, vol. 27, no. 20, pp. 2915-2916, 2011.

[38] D. Szklarczyk, A. Franceschini, S. Wyder et al., "STRING v10: protein-protein interaction networks, integrated over the tree of life," Nucleic Acids Research, vol. 43, no. D1, pp. D447D452, 2015.
[39] P. Sneha, D. Thirumal Kumar, C. George Priya Doss, R. Siva, and H. Zayed, "Determining the role of missense mutations in the POU domain of HNF1A that reduce the DNA-binding affinity: a computational approach," PLoS One, vol. 12, no. 4, article e0174953, 2017.

[40] M. Desai and J. B. Chauhan, "Computational analysis for the determination of deleterious nsSNPs in human MTHFR gene," Computational Biology and Chemistry, vol. 74, pp. 20 30, 2018.

[41] R. G. Walker, X. Deng, J. T. Melchior et al., "The structure of human apolipoprotein A-IV as revealed by stable isotopeassisted cross-linking, molecular dynamics, and small angle x-ray scattering," Journal of Biological Chemistry, vol. 289, no. 9, pp. 5596-5608, 2014.

[42] J. P. Segrest, M. K. Jones, A. Catte, and S. P. Thirumuruganandham, "Validation of previous computer models and MD simulations of discoidal HDL by a recent crystal structure of apoA-I," Journal of Lipid Research, vol. 53, no. 9, pp. 1851$1863,2012$.

[43] T. R. Caulfield, "Inter-ring rotation of apolipoprotein A-I protein monomers for the double-belt model using biased molecular dynamics," Journal of Molecular Graphics and Modelling, vol. 29, no. 8, pp. 1006-1014, 2011.

[44] Z. P. Parra-Guillen, J. Fioravanti, J. Medina-Echeverz et al., "Kinetic and dynamic computational model-based characterization of new proteins in mice: application to interferon alpha linked to apolipoprotein A-I," PLoS One, vol. 7, no. 7, article e42100, 2012.

[45] A. Gassoum, N. E. Abdelraheem, and N. Elsadig, "Comprehensive analysis of rsSNPs associated with hypertension using in-silico bioinformatics tools," Open Access Library Journal, vol. 3, no. 7, article e2839, 2016.

[46] I. N. Holcomb, R. C. Kabakoff, B. Chan et al., "FIZZ1, a novel cysteine-rich secreted protein associated with pulmonary inflammation, defines a new gene family," The EMBO Journal, vol. 19, no. 15, pp. 4046-4055, 2000.

[47] C. M. Steppan, E. J. Brown, C. M. Wright et al., "A family of tissue-specific resistin-like molecules," Proceedings of the National Academy of Sciences of the United States of America, vol. 98, no. 2, pp. 502-506, 2001. 


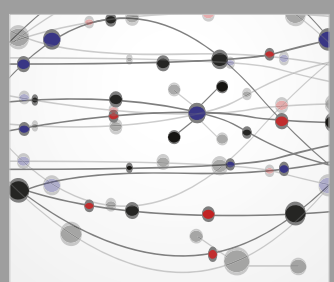

The Scientific World Journal
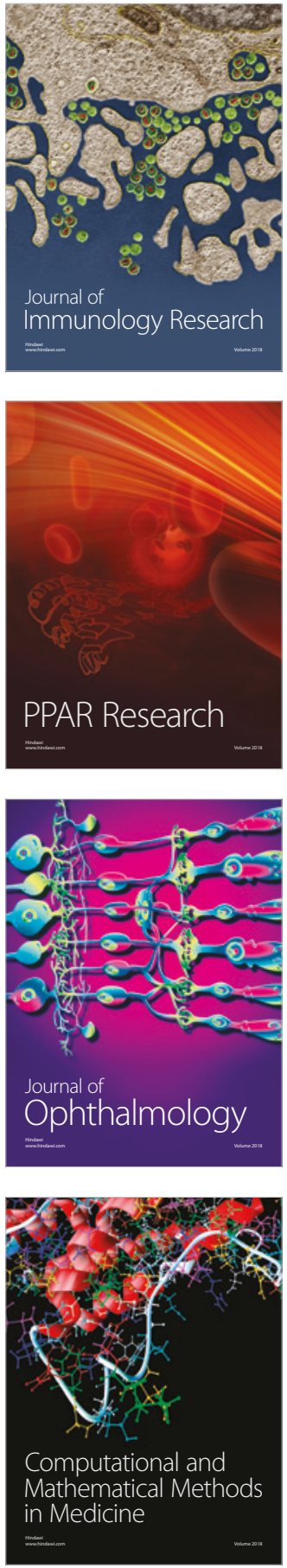

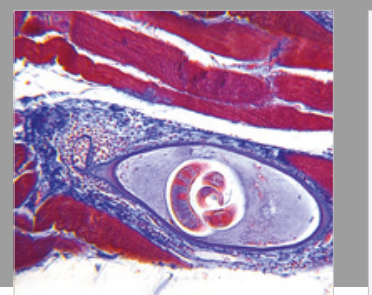

Gastroenterology Research and Practice

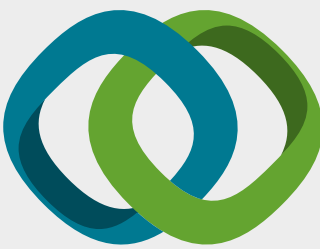

\section{Hindawi}

Submit your manuscripts at

www.hindawi.com
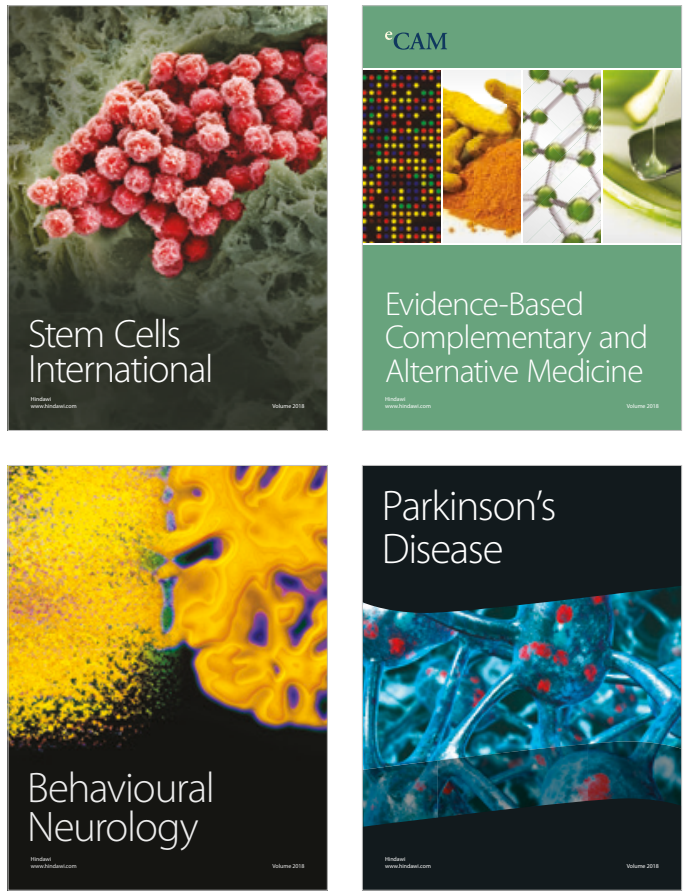

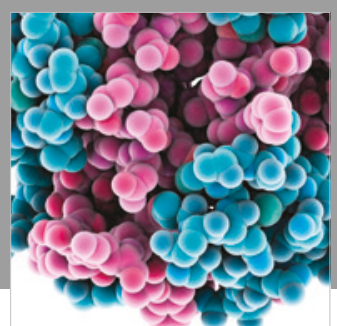

ournal of

Diabetes Research

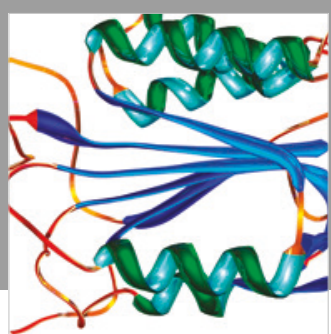

Disease Markers
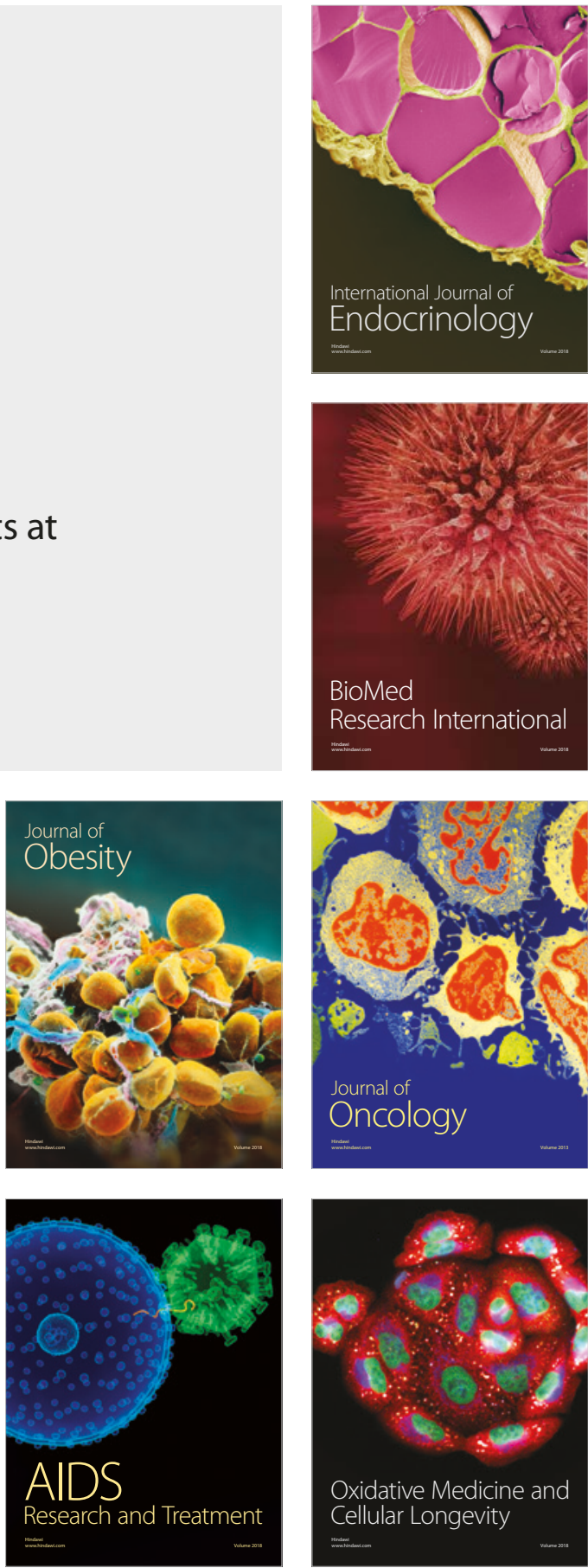